\title{
Angiotensin converting enzyme 2 in coronavirus disease (COVID-19): Evidence from bioinformatics analysis
}

\section{Xiaomei Yang ${ }^{\mathrm{a}}$, HongJin Zhao ${ }^{\mathrm{b}}$, Yan Li ${ }^{\mathrm{c}}$, Peng Zhang a*}

a. Department of Anesthesiology, Qilu Hospital of Shandong University, Jinan, Shandong, China, 250012

b. Department of Cardiology, Shandong Provincial Hospital, Jinan, Shandong Province, China, 250021

c. School of Medicine, Shandong University, Jinan, Shandong Province, China, 250012

Address all correspondence and requests for reprints to:

*. Corresponding Author. Phone: +8618560083816 ;

Email: Peng.zhang@email.sdu.edu.cn

Postal address: Department of Anesthesiology, Qilu Hospital of Shandong University, No. 107 Wenhua Xi Road, Jinan, Shandong, China, 250012

\begin{abstract}
Recently, the outbreak of coronavirus disease 2019 (COVID-19) is threatening human health globally. There is a dire need to find potential therapeutic agents. Angiotensin converting enzyme 2 (ACE2), as an entry receptor of severe acute respiratory syndrome coronavirus 2 (SARS-CoV-2), is considered as potential therapeutic target in COVID-19 pandemic. Here, our bioinformatics analysis revealed that the biological function of ACE2 was correlated with regulation of blood pressure and mediation of SARS-CoV-2 entry into host cells. Ten ACE2 cooperative proteins were identified by using STRING with a high score. ACE2 expressed highly in the small intestine, testis, and kidney. The level of ACE2 expression in tumor tissues varies in different types of cancers compared with that in normal tissues. It was worth noting that the expression level of ACE2 in the tumor has no effect on patient survival. MiRNA hsa-miR942-5p, and three transcription factors (TFs) including Signal transducer and activator of transcription 4 (STAT4), Estrogen related receptor a (ESRRA), and Signal transducer and activator of transcription 3 (STAT3) were selected as novel ACE2 regulators. Moreover, nine
\end{abstract}


potential therapeutic drugs were predicted by two online databases. Thus, our research may expand the overall view of ACE2 in COVID-19 treatment.

Key words: COVID-19; Coronavirus; ACE2; bioinformatics analysis; drug prediction

\section{Introduction}

The spread of COVID-19 is progressing rapidly. The high lethality of COVID-19, and its enormous economic and social impact, makes it paramount for us to battle this virus. Numerous therapeutic strategies have been applied to treat or block the spread of COVID-19.1 So far, there have been no specific drugs or methods identified to prevent the spread of the virus or to treat patients. 2 Intensive research on the coronavirus is needed to elucidate its pathogenic mechanisms. SARS-CoV-2 is a member of the $\beta$-coronavirus family, which includes the severe acute

respiratory syndrome coronavirus (SARS-CoV) and the Middle East respiratory syndrome coronavirus (MERS-CoV).3 In 2003, ACE2 in host cells was confirmed as the entry receptor of SARS-CoV4. Based on the similarity of the spike proteins between SARS-CoV and SARS-CoV2, SARS-CoV-2 is also expected to bind to ACE2 as its cellular entry receptor.5 Therefore, ACE2-expressing cells and tissues potentially act as targets of the novel coronavirus. Furthermore, the binding affinity between ACE2 and SARS-CoV-2 is approximately 10- to 20fold higher than that between ACE2 and SARS-CoV,6 which might contribute to its quick transmission.

ACE2, a homolog of angiotensin-converting enzyme (ACE), is a transmembrane protein including an extracellular domain, transmembrane region, and intracellular component.7 The best-known function of ACE2 is to catalyze angiotensin II to angiotensin 1-7, which dilates the arterial vessels, counteracting the adverse action of the renin-angiotensin system.8 It was reported previously that ACE2 has a protective role in severe acute lung injury and reduces the vascular permeability and lung edema induced by acid aspiration.9 Moreover, loss of ACE2 increases urinary albumin excretion and is associated with glomerular lesions and more severe renal injury.10 ACE2 regulates innate immunity and microbial ecology in the intestine, and a deficiency of ACE2 increases the susceptibility to intestinal inflammation induced by epithelial damage. 11 
Therefore, ACE2 might become a potential target molecule for COVID-19 from the pharmacological perspective.12 Presently, many supportive care plans and non-specific drugs are recommended to improve the symptoms of infected patients. 13 However, few drugs or methods have been developed targeting the ACE2 receptor on host cells. Integrative bioinformatics methods for analyzing public data have been extensively applied to unravel reliable and precise clues for further research.14 By re-analyzing the public online data of ACE2, our study intends to offer a comprehensive atlas of ACE2 expression in the different tissues and diseases and shed a favorable light on potential treatments for COVID-19.

Currently, our research data provides the following suggestions for prevention and treatment of COVID-19. First, we can partially explain the symptoms of COVID-19 and the high comorbidities in hospitalized patients. Also, it was shown in our study that the expression level of ACE2 has no effect on cancer patients' survival. However, we suggest that more attention should be paid to individuals with cancer and impaired immune systems during the outbreak of COVID-19. Second, miRNA hsa-miR-942-5p, and the transcription factors STAT4, ESRRA, and STAT3, could be useful regulators for ACE2 expression. Also, nine drugs were identified as potential therapeutic medicine for COVID-19.

\section{Methods}

\subsection{ACE2 expression analysis}

To explore the biological function of ACE2, gene ontology (GO) enrichment was performed by DAVID version 6.8. Proteins with a high combined score with ACE2 identified with the STRING online database were selected to search for proteins associated with ACE2. The protein-protein interaction (PPI) network and co-expression analysis of ACE2 and associated proteins were constructed by the STRING online database. The GO and Kyoto Encyclopedia of Genes and Genomes (KEGG) pathway enrichment analysis generated by STRING were visualized by the ggplot2 package in R (3.6.2) software.

\subsection{Tissues ACE2 expression analysis}

The Human Protein Atlas (https://www.proteinatlas.org/), a human protein database, was used to map the expression of ACE2 in normal tissues. Gene-Cloud of Biotechnology Information (GCBI, https://www.gcbi.com.cn), a genetic and data combination platform, was 
used to analyze the expression of ACE2 in different diseases. Gene Expression Profiling Interactive Analysis (GEPIA, http://gepia2.cancer-pku.cn), a gene expression analysis web server, was used to perform the survival analysis and determine the prognostic value of ACE2 in cancer patients.

\subsection{Prediction of miRNAs for ACE2}

To screen for miRNAs that modulate ACE2 gene expression, three online databases, DIANA (http://diana.imis.athena-innovation.gr/DianaTools/index.php?r=microT_CDS/index), miRWalk (http://mirwalk.uni-hd.de/), and starBase (http://starbase.sysu.edu.cn) were used to explore the miRNAs of ACE2. MiRNAs that could be predicted by all three databases were considered as ACE2-correlated miRNAs.

\subsection{Prediction of transcription factors for ACE2 and its associated genes}

To understand the transcriptional mechanism involved in the modulation of ACE2 and its associated genes, the transcription factors were predicted by the iRegulon app in Cytoscape. In this study, the criteria for prediction of TFs was the following: the minimum identity between orthologous genes was 0.05 , the maximum false discovery rate (FDR) on motif similarity was equal to 0.001 , and targets $>5$ and the NES $>4.5$ were set as the threshold. The network was established with transcription factors-genes and visualized with Cytoscape.

\subsection{Drug-target interactions}

ACE2 served as the potential target for searching for drugs through DrugBank (https://www.drugbank.ca/) and the drug-gene interaction database (DGIdb, http://www.dgidb.org/). DrugBank serves as a bioinformatics and cheminformatics resource combining detailed drug data with comprehensive drug target information.15 DGIdb mines druggene interactions and the druggable genome from papers, databases, and web resources.16

\section{Results}

\subsection{ACE2 expression analysis}

Our data indicated that the GO analysis of ACE2, which is mainly enriched in angiotensin maturation and regulation of systemic arterial blood pressure by renin-angiotensin, and at the same time we can see that ACE2 has a role in inflammatory responses and mediates entry of the 
virus into host cells (Table 1). The cellular component enrichment showed that the protein ACE2 was composed of three parts: an extracellular region, components of the membrane, and plasma components. Its molecular function is mainly enriched in virus receptor activity, glycoprotein binding, endopeptidase activity, and carboxypeptidase activity. Furthermore, KEGG pathway enrichment suggested that the protein ACE2 is involved in the pathway of the renin-angiotensin system, and the protein digestion and absorption pathways.

Ten proteins (Type-1 angiotensin II receptor (AGTR1), Type-2 angiotensin II receptor (AGTR2), Angiotensinogen (AGT), Renin (REN), Neprilysin (MME), Lysosomal Pro-X carboxypeptidase (PRCP), Dipeptidyl peptidase 4 (DPP4), Meprin A subunit alpha (MEP1A), Meprin A subunit beta (MEP1B), and Xaa-Pro aminopeptidase 2 (XPNPEP2)) were identified by STRING with scores $>0.85$ (Figure $1 \mathrm{~A}$ and $\mathrm{B}$ ). The GO enrichment analysis of these proteins was categorized according to biological process (BP), molecular function (MF), cellular component (CC), and KEGG pathway as shown in (Figure $2(\mathrm{~A} \sim \mathrm{D})$ ). Furthermore, the biological process was enriched in regulation of systemic arterial blood pressure by renin-angiotensin and regulation of blood volume by renin-angiotensin, which indicates that these 11 proteins are mainly responsible for regulating blood pressure. Meanwhile, the molecular function enrichment revealed that these proteins are not only different proteases like peptidase and metallopeptidase, but also have virus-binding functions. The cellular component enrichment showed they are membrane proteins like ACE2, including an extracellular region, a membrane component, and a plasma membrane part. By examining the KEGG pathway enrichment, we found that these proteins are mainly enriched in the renin-angiotensin system and protein digestion and absorption, which is similar to the ACE2 protein pathway enrichment.

\subsection{Tissues ACE2 expression analysis}

The expression levels of ACE2 protein and mRNA in normal tissues are shown in Figure $3 \mathrm{~A}$ and B. Our data indicate that ACE2 is highly expressed in the small intestine, kidney, and testis, which indicates that there are high levels of ACE2 in the digestive system, urinary system, and genital system. GCBI online databases were searched to explore the expression of ACE2 in different diseases. As shown in Figure 3C, research related to ACE2 is mainly related to hypertension, severe acute respiratory syndrome, diabetes mellitus, kidney diseases, and cardiovascular diseases. 
The gene expression differences between tumor and normal control tissue indicated that among these 33 cancers, some cancers like adrenocortical carcinoma (ACC), kidney renal papillary cell carcinoma (KIRP), and kidney renal clear cell carcinoma (KIRC) express higher levels of ACE2 than the normal tissues (Fig 3D). Other cancers such as stomach adenocarcinoma (STAD), sarcoma (SARC), and kidney chromophobe (KICH) express lower levels of ACE2 than normal tissues. To understand the role of ACE2 in cancers, we explored the overall survival analysis through the GEPIA online database as shown in Fig 4A J. The results showed that there are 4740 ACE2 higher expression samples and 4738 lower expression samples among 33 cancers. There is no obvious difference in overall survival rate between the two groups (Fig 4A). Further analysis showed that the expression level of ACE2 had no effect on patients' survival in higher level cancers like ACC and KIRP (Fig 4B and C) or in the lower level cancers like KICH, SARC, and STAD (Fig 4H J). Among the 33 types of tumors, the expression of ACE2 had an obvious effect only for four cancers, KIRC, brain Lower Grade Glioma (LGG), liver hepatocellular carcinoma (LIHC), and ovarian serous cystadenocarcinoma $(\mathrm{OV})$. It is worth mentioning that high ACE2 increased the overall survival of patients with KIRC, LIHC, and OV, but decreased the overall survival rate of patients with LGG.

\subsection{Prediction of miRNAs}

The miRNAs of ACE2 that could be predicted in all three databases were identified as potential miRNAs. We found 60 miRNAs were predicted by DIANA, 180 miRNAs were predicted by miRwalk, 13 miRNAs were predicted by starBase, and 9 miRNAs were predicted by two databases. Only one miRNA, hsa-miR-942-5p, was predicted by all three databases as indicated in Fig 5B.

\subsection{Prediction of transcription factors}

As shown in Fig 5A, three transcription factors, STAT4, ESRRA, and STAT3, were predicted by iRegulon plugin in Cytoscape. STAT4 is linked to 6 genes, ESRRA is linked to 7 genes, and STAT3 is linked to 8 genes. ACE2 is targeted by all three transcription factors.

\subsection{Drug-target interactions}

Using ACE2 to explore drug protein interactions, nine promising drugs were compiled and selected from two databases (Table 2). The drugs approved by the FDA are lisinopril, ramipril, 
chloroquine, and hydroxychloroquine. Lisinopril and ramipril are angiotensin converting enzyme inhibitors (ACEIs) and block the contact of angiotensin II with angiotensin converting enzyme by dilating constricted blood vessels ${ }^{17}$. Chloroquine and its derivative hydroxychloroquine are used to treat malaria, and are receiving increased attention due to their anti-inflammatory, immunomodulating, and antitumoral effects ${ }^{18}$.

\section{Discussion}

To explore the role of ACE2 in COVID-19, this research is, for the first time, using a bioinformatics method to explore the biological function of ACE2, reveal the expression of ACE2 in different tissues, explain the role of ACE2 in different diseases, and predict possible miRNA, transcription factors, and drugs targeting ACE2.

The biological process analysis demonstrated that ACE2 is involved in the regulation of cytokine production, angiotensin maturation, and regulation of systemic arterial blood pressure by renin-angiotensin, and at the same time, it was shown in our study that ACE2 plays an essential role in inflammatory responses and mediates the viral entry into host cells. The molecular function enrichment revealed that ACE2 can bind to the glycoprotein of the virus receptor, and act as a protease, like endopeptidase, carboxypeptidase, and metallocarboxypeptidase. Furthermore, it was shown by KEGG pathway enrichment that ACE2 takes part in the renin-angiotensin system and protein digestion and absorption. As a transmembrane protein, ACE2 catalyzes angiotensin II to angiotensin 1-7, and also hydrolyzes apelin-13 and dynorphin A 1-13.19 ACE2 and its product angiotensin 1-7 have multiple effects such as vasodilation, anti-hypertension, and inhibition of cardiac remodeling, and counteract against the adverse action of the renin-angiotensin system (RAS). 8 As the entrance receptor, ACE2 can bind to the spike protein of SARS and SARS-CoV-2, and it mediates the virus's entry into host cells. 4,5

Considering the potential cooperation of other proteins with ACE2 on the cell membrane, the protein-protein interaction network of ACE2 and its associated proteins was constructed by using the STRING online database. Gene ontology annotations related to ACE2 and its associated proteins are mainly related to regulation of systemic arterial blood pressure by reninangiotensin and regulation of systemic arterial blood pressure, which are similar to the biological function analysis of ACE2. Among the ten associated proteins, AGT is a potent regulator of 
blood pressure and belongs to the vital component of the renin-angiotensin system (RAS).20 REN cleaves AGT in plasma into angiotensin I and initiates the cascade of RAS.21 As the different types of angiotensin II receptors, AGTR1 mediates the vasopressor effect of angiotensin II 22 while AGTR2 inhibits cell proliferation and dilates blood vessels.23

As different members of the peptidase M12A family, MEP1A and MEP1B hydrolyze proteins and peptides, and are involved in cancer and inflammation processes. MME, one member of the M13 family, cleaves angiotensin I to Ang (1-7) and angiotensin II to degradation products.24 PRCP as a prolylcarboxypeptidase cleaves the C-terminal amino acids of angiotensin II, III, and des-Arg9-bradykinin.25 XPNPEP2 hydrolyzes the N-terminal of proteins such as vasodilator kinin and is helpful for screening patients susceptible to adverse reactions associated with angiotensin-converting enzyme inhibitors.26 DPP4, a cell surface glycoprotein receptor, positively regulates $\mathrm{T}$ cell coactivation and can be activated by angiotensin II concurrent with kidney injury. 27

The map of ACE2 protein and mRNA expression in tissues might give us some clues to predict the symptoms and consequences of COVID-19. Patients with infection may present with nausea, vomiting, and loose stool very early in the disease progression,28 suggesting that the digestive system might be affected directly by viral infection. This is consistent with our finding that ACE2 is highly expressed in the small intestine. The high expression of ACE2 in tissues such as the kidney reminds us of the threat to patients with renal dysfunction or on dialysis. Yang et al have reported a high prevalence of acute kidney impairment in hospitalized COVID-19 patients.29 SARS infection can cause testis inflammation,30 but the damage of SARS-CoV-2 to the testicle is not clear. The reproductive function should be followed and monitored in recovered male COVID-19 patients because of the high expression level of ACE2 in the testis. At the same time, the low level of ACE2 in the lung may partially explain why hypoxia and dyspnea are not so obvious in patients within the early infection stage.

In one clinical study that included 1099 patients with COVID-19, a high incidence of comorbidities such as hypertension, diabetes mellitus, coronary heart diseases, and cerebrovascular diseases was confirmed in severely infected patients. $31 \mathrm{In}$ another study, 30\% of hospitalized patients with COVID-19 had hypertension and $12 \%$ of them had diabetes mellitus.32 Moreover, a third clinical study showed that the patients in hospital receiving 
intensive care were more likely to have underlying comorbidities, including hypertension, diabetes, cardiovascular disease, cerebrovascular disease, and malignancies.33 Notably, the most frequent comorbidities reported in the three studies are hypertension and diabetes mellitus. Consistent with these clinical findings, our study showed that the pathways related to ACE2 are mainly involved in hypertension, severe acute respiratory syndrome, diabetes mellitus, kidney diseases, and cardiovascular diseases. ACE2 has a protective role in cardiac contraction, arterial vessel dilation, respiratory system inflammation, and kidney injury.34 The coronavirus infection blocks the protective role of ACE2 in the circulatory and respiratory systems. Thus, the above findings indicate that patients with cardiopulmonary and cerebrovascular comorbidities are in need of more attention and care.

To understand the role of ACE2 in cancers, we explored the overall survival through the GEPIA online database and found various cancers express ACE2 at different levels, rendering them potential targets of COVID-19. The results revealed that there were 4740 ACE2 higher expression samples and 4738 lower expression samples among 33 types of tumors. There was no obvious difference in overall survival between the two groups. Further analysis showed that the expression of ACE2 has no effect on patients' survival in higher level groups like ACC and KIRP, or in the lower level groups like KICH, SARC, and STAD. However, we advise that attention should be given to individuals with KIRC, LIHC, or OV since our research showed that high expression of ACE2 is positively correlated with the survival of these cancer patients. There is a challenge for these patients when the viruses are occupying the ACE2 receptor. As for LGG patients, there is no research to prove they may benefit from the virus infection although a low expression of ACE2 prolongs these patients' survival time. The immune system of cancer patients is suppressed after surgery, chemotherapy, or radiotherapy.35,36,37 The high incidence of infected cancer patients in the ICU might be attributable to their impaired immune system before virus infection. 38

Previous research proved that the virus spreads from person to person and not in mice because of the different structure of ACE2, which highlights the vital role of ACE2 in COVID19.39 Given the important role of ACE2 in coronavirus invading host cells, identification of ACE2 regulators is important for prevention and treatment of virus infection. 
MicroRNAs are small and non-coding RNA molecules that are known to regulate protein translation or targeted mRNA cleavage.40 Numerous studies have highlighted miRNAs as potential therapeutic targets. In our study, hsa-miR-942-5p was predicted by three databases. A previous study indicated that miR-942-5p is related to Kaposi's sarcoma-associated herpesvirus (KSHV). High miR-942-5p expression inhibits KSHV replication by targeting the inhibitor of

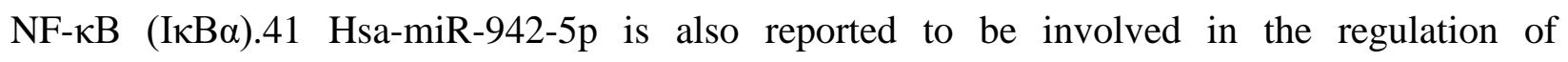
cytochrome P4502E1 in the liver.42 In the development of chronic thromboembolic pulmonary hypertension, hsa-miR-942-5p connects with cirRNA has_circ_0002062 and the gene CDK6 to form a network regulating the cell cycle and proliferation.43 Our findings suggested that hsamiR-942-5p could play a noticeable role in the development and prognosis of COVID-19 through regulating the disease-associated ACE2 receptor.

Transcription factors bind to specific genes and control multiple biological responses, and are regarded as potential drug targets. 44 The estrogen related receptor $\alpha$ (ESRRA), an orphan nuclear receptor, regulates energy homeostasis and innate host defense. ESRRA activates autophagy to promote the innate antimicrobial defense against mycobacterial infection, protecting against mycobacterial infection.45 Moreover, signal transducer and activator of transcription 3 (STAT3), as a transcription factor, is associated with inflammation, survival, and invasion.46 STAT3 activates transcription in the nucleus, regulates the phosphorylation of p38 mitogen-activated protein kinase, and reduces the cytopathic effects of SARS-CoV infection, which exhibits an antivirus function.47 Also, signal transducer and activator of transcription 4 (STAT 4), another member of the STAT family, is implicated in immune responses against pathogens and inflammatory disorders. STAT4 is stimulated by cytokines and consequently drives $\mathrm{T}$ helper cells differentiation, modulating autoimmune and inflammatory reactions.48 Coincident with previous studies, our research predicted that the transcription factors STAT4, ESRRA, and STAT3 were regulators of ACE2 and its associated proteins, and might be possible therapeutic opportunities against the coronavirus infection through modulating the transcriptional activation of ACE2.

During the outbreak of COVID-19, patients with cardiovascular diseases are suffering more than healthy people. It has been reported that angiotensin converting enzyme inhibitors (ACEIs) improve the clinical outcomes and decrease inflammation in COVID-19 patients with 
hypertension.49 ACEIs block ACE from converting angiotensin I to angiotensin II. Increased angiotensin I is catalyzed by ACE2 to angiotensin 1-9 and subsequently angiotensin 1-7 by ACE.50 Angiotensin 1-7 is recognized as playing a key role in anti-hypertension and antiinflammatory reactions, 51 which is regarded as mediating one of the beneficial effects of ACEIs on COVID-19 hypertension patients.50 In our research, ACEIs were identified as inhibitors of ACE2, a homolog of ACE, by two online databases, though we did not find experiments to support these results. We supposed that the beneficial effects of ACEIs on COVID-19 with hypertension might partially be due to ACE2, the entry receptor of the virus, being partially occupied by ACEIs and high levels of angiotensin I, and thus fewer viruses can enter into the host cells.

Chloroquine and hydroxychloroquine are anti-malaria drugs in use for decades.18 In 2005, chloroquine was proven to interfere with the terminal glycosylation of ACE2, abrogate SARS$\mathrm{CoV}$ binding with its receptor, and prevent the spread of SARS-CoV.52 In the pandemic of COVID-19, chloroquine was reported to effectively block SARS-CoV-2 infection in China. The anti-COVID-19 mechanism of chloroquine is believed to be related to its regulation of ACE2 glycosylation and the host immune response.53 However, the chloroquine treatment is controversial, the cautiousness is mainly related to its poor clinical studies and toxicological risks as retinopathy, cardiomyopathy and infectious mononucleosis.54,55 As for the effect of whether an ACE2 agonist or post-translational modifications as acetylation, phosphorylation and ubiquitination would facilitate SARS-CoV-2 infection or not, is also an interesting question that need further investigation.

\section{Conclusion}

Currently, COVID-19 is spreading rapidly and has thrown the whole world into a panic. Intense investigation into the molecular mechanisms of the virus receptor ACE2 is essential for its prevention, diagnosis, and therapy. In this study, we identified the biological function of ACE2, partially explained the clinical manifestations and comorbidities of COVID-19, and predicted miRNA hsa-miR-942-5p, transcription factors STAT4, ESRRA, STAT3, and nine targeted drugs aimed at ACE2 by using bioinformatics methods. Further in-depth experiments are needed to confirm the rational therapy for treating COVID-19.

\section{Conflict of interest}


The authors declare no conflicts of interest.

\section{Acknowledgements}

This work was supported by Shandong Provincial Natural Science Foundation of China (Grant number ZR2015PH036, ZR2014HM027) and China Scholarship Council (No.201706225023).

\section{Author contributions}

Peng Zhang designed the study. Xiaomei Yang performed the analysis and wrote the manuscript.

Hongjin Zhao and Yan Li participated in the analysis. All authors reviewed and approved the final manuscript.

\section{References}

1. Yang Y, Islam MS, Wang J, Li Y, Chen X. Traditional Chinese Medicine in the Treatment of Patients Infected with 2019-New Coronavirus (SARS-CoV-2): A Review and Perspective. Int J Biol Sci. 2020;16(10):1708-1717.

2. Li G, De Clercq E. Therapeutic options for the 2019 novel coronavirus (2019-nCoV). Nat Rev Drug Discov. 2020;19:149-150.

3. Fehr AR, Perlman S. Coronaviruses: an overview of their replication and pathogenesis. Methods in molecular biology. 2015;1282:1-23.

4. Li W, Moore MJ, Vasilieva N, et al. Angiotensin-converting enzyme 2 is a functional receptor for the SARS coronavirus. Nature. 2003;426:450-454.

5. Zhou P, Yang XL, Wang XG, et al. A pneumonia outbreak associated with a new coronavirus of probable bat origin. Nature. 2020;579:270-273.

6. Wrapp D, Wang N, Corbett KS, et al. Cryo-EM structure of the 2019-nCoV spike in the prefusion conformation. Science. 2020;367:1260-1263.

7. Kuba K, Imai Y, Penninger JM. Multiple functions of angiotensin-converting enzyme 2 and its relevance in cardiovascular diseases. Circ J. 2013;77:301-308.

8. Jiang F, Yang J, Zhang Y, et al. Angiotensin-converting enzyme 2 and angiotensin 1-7: novel therapeutic targets. Nat Rev Cardiol. 2014;11:413-426.

9. Imai Y, Kuba K, Rao S, et al. Angiotensin-converting enzyme 2 protects from severe acute lung failure. Nature. 2005;436(7047):112-116.

10. Soler MJ, Wysocki J, Batlle D. Angiotensin-converting enzyme 2 and the kidney. Exp Physiol. 2008;93:549-556.

11. Hashimoto T, Perlot T, Rehman A, et al. ACE2 links amino acid malnutrition to microbial ecology and intestinal inflammation. Nature. 2012;487:477-481.

12. Hoffmann M, Kleine-Weber H, Schroeder S, et al. SARS-CoV-2 Cell Entry Depends on ACE2 and TMPRSS2 and Is Blocked by a Clinically Proven Protease Inhibitor. Cell. 2020.

13. Cascella M, Rajnik M, Cuomo A, Dulebohn SC, Di Napoli R. Features, Evaluation and Treatment Coronavirus (COVID-19). StatPearls. Treasure Island (FL)2020. 
14. Gligorijevic V, Malod-Dognin N, Przulj N. Integrative methods for analyzing big data in precision medicine. Proteomics. 2016;16:741-758.

15. Wishart DS, Feunang YD, Guo AC, et al. DrugBank 5.0: a major update to the DrugBank database for 2018. Nucleic Acids Res. 2018;46:D1074-D1082.

16. Cotto KC, Wagner AH, Feng YY, et al. DGIdb 3.0: a redesign and expansion of the druggene interaction database. Nucleic Acids Res. 2018;46:D1068-D1073.

17. Piepho RW. Overview of the angiotensin-converting-enzyme inhibitors. Am J Health Syst Pharm. 2000;57 Suppl 1:S3-7.

18. Plantone D, Koudriavtseva T. Current and Future Use of Chloroquine and Hydroxychloroquine in Infectious, Immune, Neoplastic, and Neurological Diseases: A Mini-Review. Clin Drug Investig. 2018;38:653-671.

19. Vickers C, Hales P, Kaushik V, et al. Hydrolysis of biological peptides by human angiotensin-converting enzyme-related carboxypeptidase. The Journal of biological chemistry. 2002;277:14838-14843.

20. Jeunemaitre X, Soubrier F, Kotelevtsev YV, et al. Molecular basis of human hypertension: role of angiotensinogen. Cell. 1992;71:169-180.

21. Moore N, Dicker P, O'Brien JK, et al. Renin gene polymorphisms and haplotypes, blood pressure, and responses to renin-angiotensin system inhibition. Hypertension. 2007;50:340-347.

22. Bergsma DJ, Ellis C, Kumar C, et al. Cloning and characterization of a human angiotensin II type 1 receptor. Biochemical and biophysical research communications. 1992;183:989-995.

23. Akishita M, Yamada H, Dzau VJ, Horiuchi M. Increased vasoconstrictor response of the mouse lacking angiotensin II type 2 receptor. Biochemical and biophysical research communications. 1999;261:345-349.

24. Banerjee S, Oneda B, Yap LM, et al. MEP1A allele for meprin A metalloprotease is a susceptibility gene for inflammatory bowel disease. Mucosal Immunol. 2009;2:220-231.

25. Tan F, Morris PW, Skidgel RA, Erdos EG. Sequencing and cloning of human prolylcarboxypeptidase (angiotensinase C). Similarity to both serine carboxypeptidase and prolylendopeptidase families. The Journal of biological chemistry. 1993;268:1663116638.

26. Molinaro G, Carmona AK, Juliano MA, et al. Human recombinant membrane-bound aminopeptidase P: production of a soluble form and characterization using novel, internally quenched fluorescent substrates. Biochem J. 2005;385:389-397.

27. Ikushima H, Munakata Y, Ishii T, et al. Internalization of CD26 by mannose 6phosphate/insulin-like growth factor II receptor contributes to T cell activation. Proceedings of the National Academy of Sciences of the United States of America. 2000;97:8439-8444.

28. Tian Y, Rong L, Nian W, He Y. Review article: Gastrointestinal features in COVID-19 and the possibility of faecal transmission. Aliment Pharmacol Ther. 2020.

29. Yang X, Yu Y, Xu J, et al. Clinical course and outcomes of critically ill patients with SARS-CoV-2 pneumonia in Wuhan, China: a single-centered, retrospective, observational study. Lancet Respir Med. 2020.

30. Xu J, Qi L, Chi X, et al. Orchitis: a complication of severe acute respiratory syndrome (SARS). Biol Reprod. 2006;74:410-416. 
31. Guan WJ, Ni ZY, Hu Y, et al. Clinical Characteristics of Coronavirus Disease 2019 in China. The New England journal of medicine. 2020.

32. Zhang JJ, Dong X, Cao YY, et al. Clinical characteristics of 140 patients infected with SARS-CoV-2 in Wuhan, China. Allergy. 2020.

33. Wang D, Hu B, Hu C, et al. Clinical Characteristics of 138 Hospitalized Patients With 2019 Novel Coronavirus-Infected Pneumonia in Wuhan, China. JAMA. 2020.

34. Pacurari M, Kafoury R, Tchounwou PB, Ndebele K. The Renin-Angiotensin-aldosterone system in vascular inflammation and remodeling. Int J Inflam. 2014;2014:689360.

35. Carvalho HA, Villar RC. Radiotherapy and immune response: the systemic effects of a local treatment. Clinics (Sao Paulo). 2018;73(suppl 1):e557s.

36. Wargo JA, Reuben A, Cooper ZA, Oh KS, Sullivan RJ. Immune Effects of Chemotherapy, Radiation, and Targeted Therapy and Opportunities for Combination With Immunotherapy. Semin Oncol. 2015;42:601-616.

37. Amodeo G, Bugada D, Franchi S, et al. Immune function after major surgical interventions: the effect of postoperative pain treatment. J Pain Res. 2018;11:1297-1305.

38. Yu J, Ouyang W, Chua MLK, Xie C. SARS-CoV-2 Transmission in Patients With Cancer at a Tertiary Care Hospital in Wuhan, China. JAMA Oncol. 2020.

39. Wu F, Zhao S, Yu B, et al. A new coronavirus associated with human respiratory disease in China. Nature. 2020;579:265-269.

40. Ling H, Fabbri M, Calin GA. MicroRNAs and other non-coding RNAs as targets for anticancer drug development. Nat Rev Drug Discov. 2013;12:847-865.

41. Yan Q, Shen C, Qin J, et al. HIV-1 Vpr Inhibits Kaposi's Sarcoma-Associated Herpesvirus Lytic Replication by Inducing MicroRNA miR-942-5p and Activating NFkappaB Signaling. Journal of virology. 2016;90:8739-8753.

42. Wang Y, Yu D, Tolleson WH, et al. A systematic evaluation of microRNAs in regulating human hepatic CYP2E1. Biochemical pharmacology. 2017;138:174-184.

43. Miao R, Wang Y, Wan J, et al. Microarray expression profile of circular RNAs in chronic thromboembolic pulmonary hypertension. Medicine (Baltimore). 2017;96:e7354.

44. Butt TR, Karathanasis SK. Transcription factors as drug targets: opportunities for therapeutic selectivity. Gene Expr. 1995;4:319-336.

45. Kim SY, Yang CS, Lee HM, et al. ESRRA (estrogen-related receptor alpha) is a key coordinator of transcriptional and post-translational activation of autophagy to promote innate host defense. Autophagy. 2018;14:152-168.

46. Aggarwal BB, Kunnumakkara AB, Harikumar KB, et al. Signal transducer and activator of transcription-3, inflammation, and cancer: how intimate is the relationship? Annals of the New York Academy of Sciences. 2009;1171:59-76.

47. Mizutani T, Fukushi S, Murakami M, et al. Tyrosine dephosphorylation of STAT3 in SARS coronavirus-infected Vero E6 cells. FEBS Lett. 2004;577:187-192.

48. O'Shea JJ, Lahesmaa R, Vahedi G, Laurence A, Kanno Y. Genomic views of STAT function in CD4+ T helper cell differentiation. Nat Rev Immunol. 2011;11:239-250.

49. Meng J, Xiao G, Zhang J, et al. Renin-angiotensin system inhibitors improve the clinical outcomes of COVID-19 patients with hypertension. Emerg Microbes Infect. 2020;9:757760.

50. $\mathrm{Li} \mathrm{G}, \mathrm{Hu} \mathrm{R}$, Zhang X. Antihypertensive treatment with ACEI/ARB of patients with COVID-19 complicated by hypertension. Hypertension research : official journal of the Japanese Society of Hypertension. 2020. 
51. Gaddam RR, Chambers S, Bhatia M. ACE and ACE2 in inflammation: a tale of two enzymes. Inflamm Allergy Drug Targets. 2014;13:224-234.

52. Vincent MJ, Bergeron E, Benjannet S, et al. Chloroquine is a potent inhibitor of SARS coronavirus infection and spread. Virol J. 2005;2:69.

53. Devaux CA, Rolain JM, Colson P, Raoult D. New insights on the antiviral effects of chloroquine against coronavirus: what to expect for COVID-19? Int J Antimicrob Agents. 2020:105938.

54. Ferner RE, Aronson JK. Chloroquine and hydroxychloroquine in covid-19. BMJ. 2020;369:m1432.

55. Pereira BB. Challenges and cares to promote rational use of chloroquine and hydroxychloroquine in the management of coronavirus disease 2019 (COVID-19) pandemic: a timely review. J Toxicol Environ Health B Crit Rev. 2020:1-5. 
Figure legends

Figure 1. PPI network and co-expression of ACE2 and associated proteins constructed by STRING.

A. The PPI network of ACE2 and associated proteins. B. The co-expression analysis of ACE2 and associated proteins. PPI, protein-protein interaction. ACE2, angiotensin converting enzyme 2.

Figure 2. The GO enrichment of ACE2 and its cooperative proteins.

A. Biological process enrichment. B. Molecular function enrichment. C. Cellular component enrichment. D. KEGG pathway enrichment. GO, gene ontology. KEGG, Kyoto Encyclopedia of Genes and Genomes.

Figure 3. Expression of ACE2 and researches related to ACE2

A. Protein expression of ACE2 in tissues. B. mRNA expression of ACE2 in tissues. C. Researches related to ACE2. D. ACE2 expression in cancers. The red bar represents cancer tissues, the blue bar represents normal control tissues.

Figure 4. The prognostic value of ACE2 in cancers in the GEPIA database.

A. Kaplan-Meier plot of ACE2 of 33 cancers. B. Kaplan-Meier plot of ACE2 for ACC. C. Kaplan-Meier plot of ACE2 for KIRP. D. Kaplan-Meier plot of ACE2 for KERC. E. KaplanMeier plot of ACE2 for LGG. F. Kaplan-Meier plot of ACE2 for LIHC. G. Kaplan-Meier plot of ACE2 for OV. H. Kaplan-Meier plot of ACE2 for KICH. I. Kaplan-Meier plot of ACE2 for SARC. J. Kaplan-Meier plot of ACE2 for STAD. GEPIA, Gene Expression Profiling Interactive Analysis. The red line is the high ACE2 group, the blue line is the low ACE2 group. HR, Hazard ratio. TPM, Transcripts per million. ACC, Adrenocortical Carcinoma. KIRP, Kidney renal papillary cell carcinoma. KIRC, Kidney renal clear cell carcinoma. LGG, Brain lower grade glioma. LIHC, Liver hepatocellular carcinoma. OV, Ovarian serous cystadenocarcinoma. KICH, Kidney chromophobe. SARC, Sarcoma. STAD, Stomach adenocarcinoma.

Figure 5. Transcription factors-ACE2 and associated proteins network and miRNAs Venn diagram. 
A. Network of transcription factors and ACE2 with its associated proteins. B. miRNAs Venn diagram of the three databases. The purple circle is the ACE2 associated proteins, the green circle is transcription factors, and the yellow circle is ACE2. The Venn diagram reveals the overlapping miRNAs created by the three databases, and the cross areas are the overlapping miRNAs.

Table 1. The GO enrichment of ACE2 by DAVID. GO, gene ontology.

Table 2. Candidate drugs targeting ACE2. 


\section{Figure 1}

A

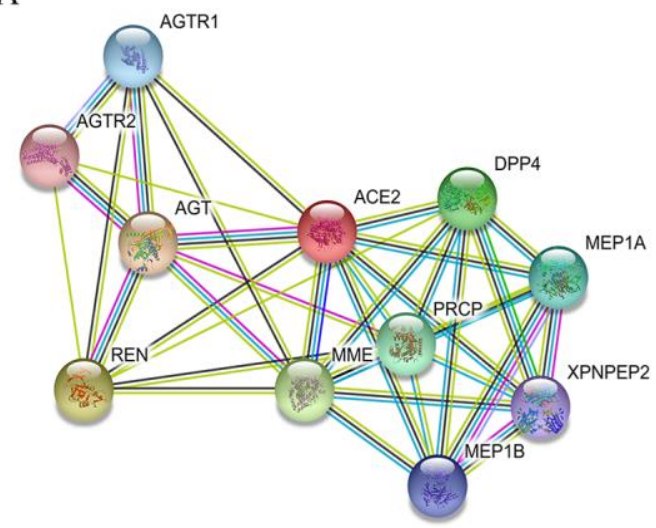

B

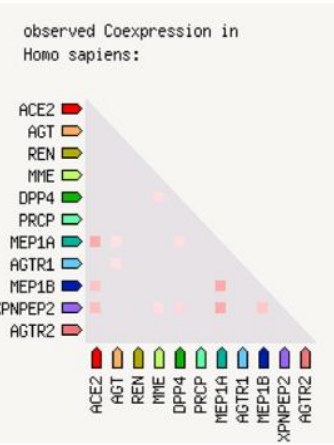

observed Coexpression in other organisms (transferred):

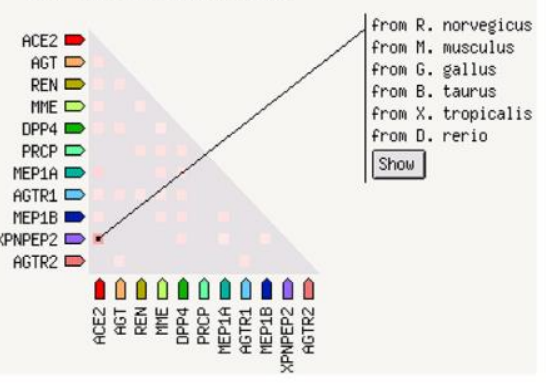


Figure 2

A

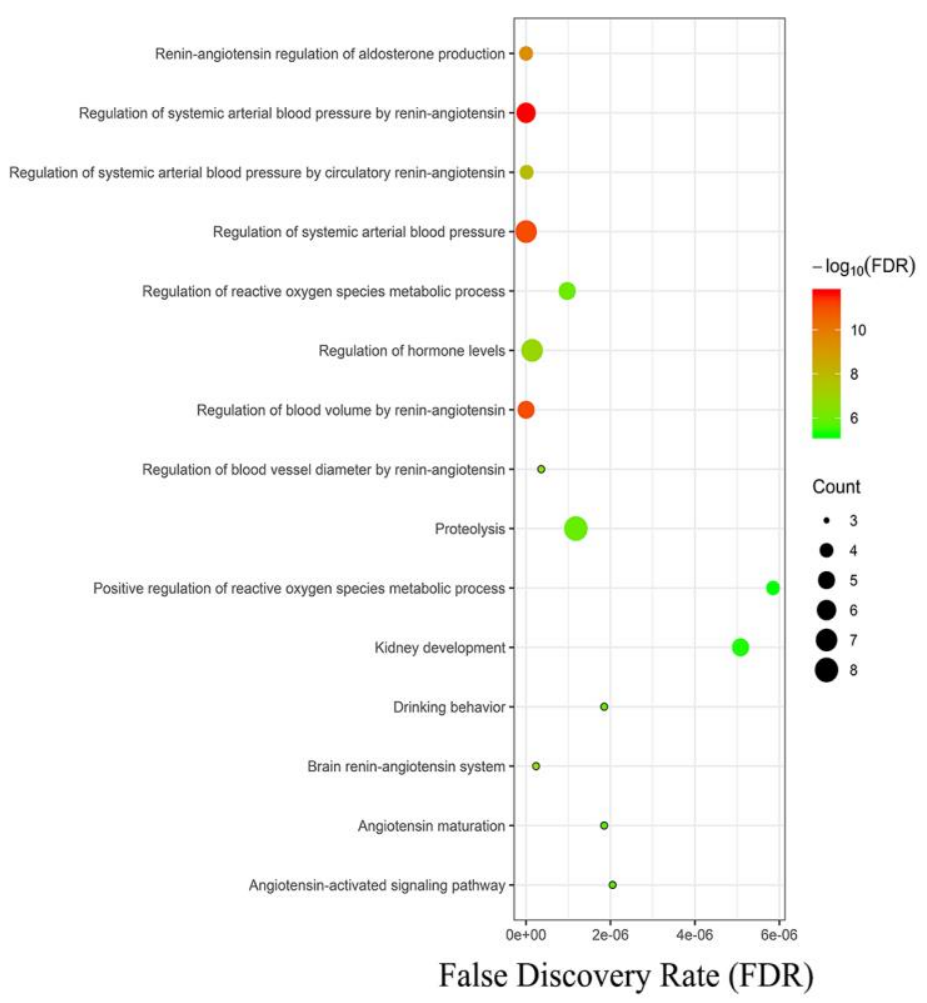

C

Cellular Component Enrichment

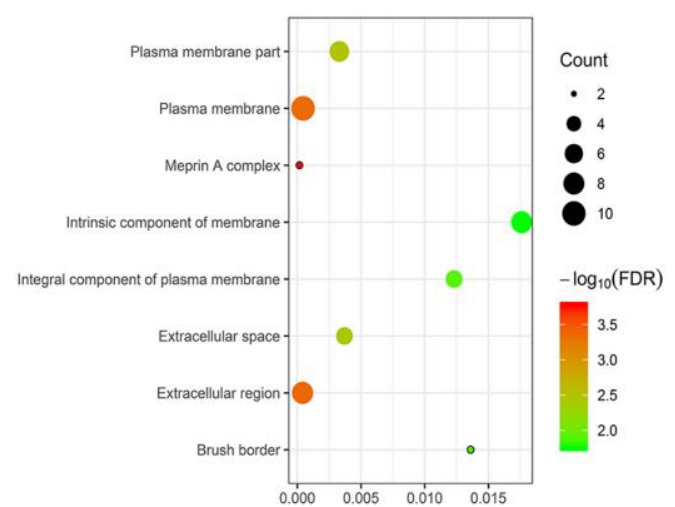

False Discovery Rate (FDR)
B Molecular Function Enrichment

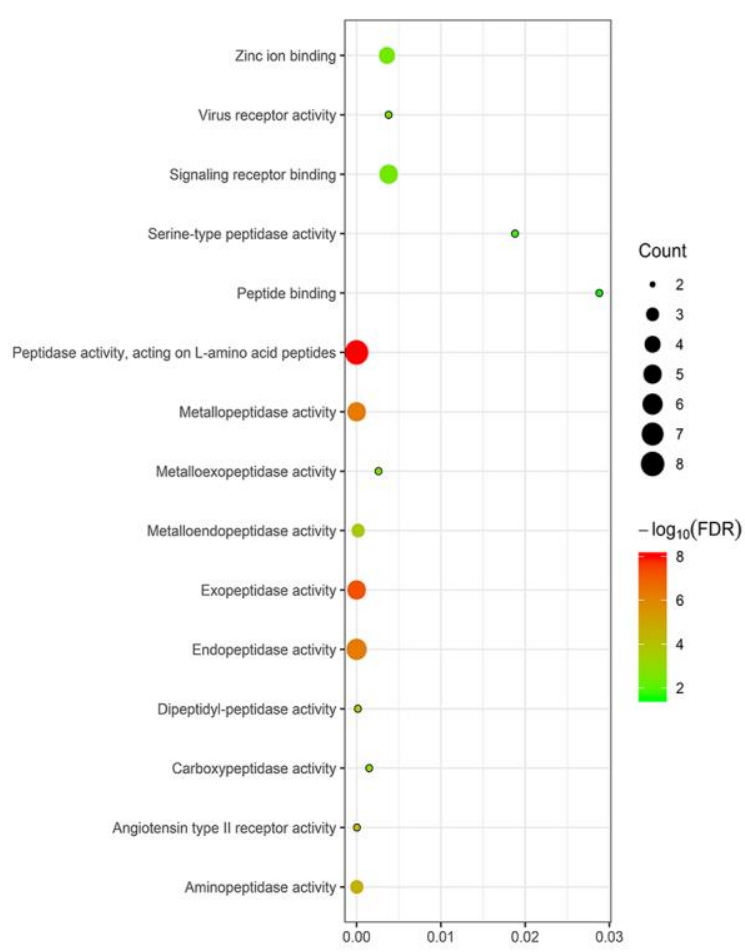

False Discovery Rate (FDR)

D

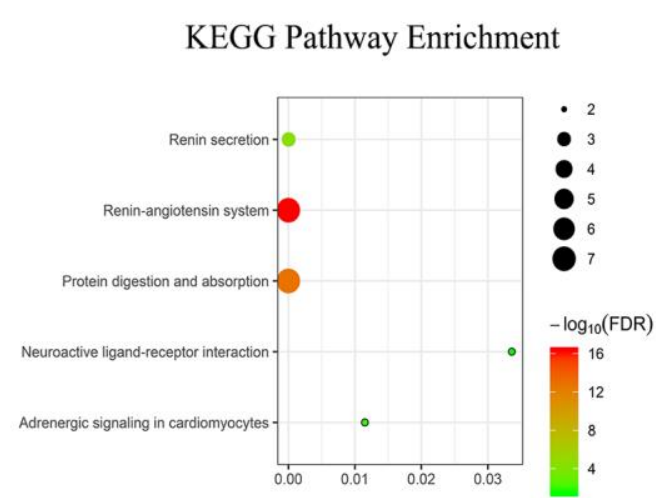

False Discovery Rate (FDR) 


\section{Figure 3}
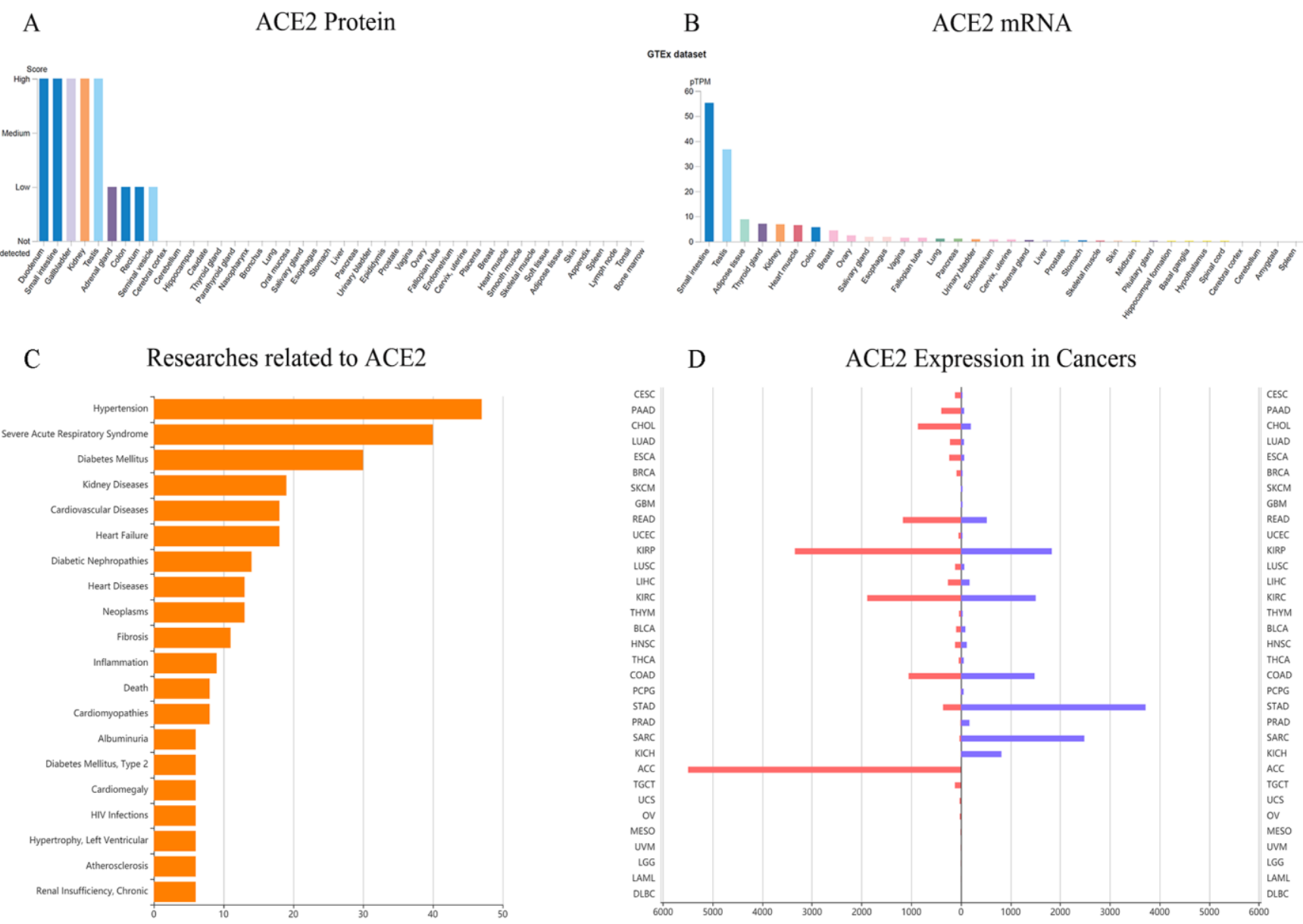

D

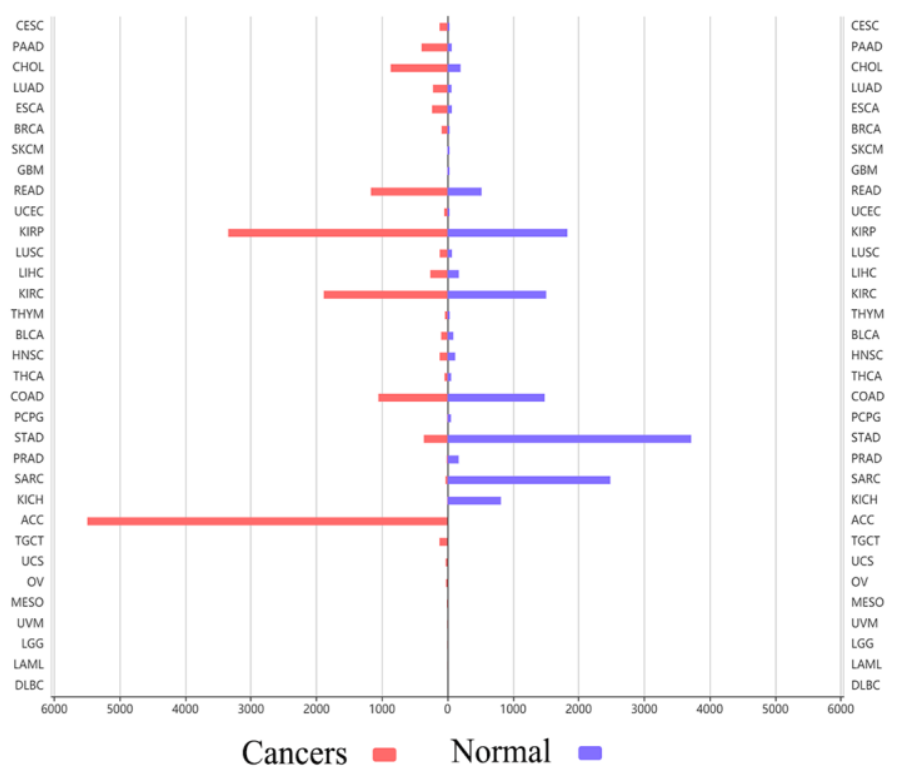

Article Numbers 


\section{Figure 4}
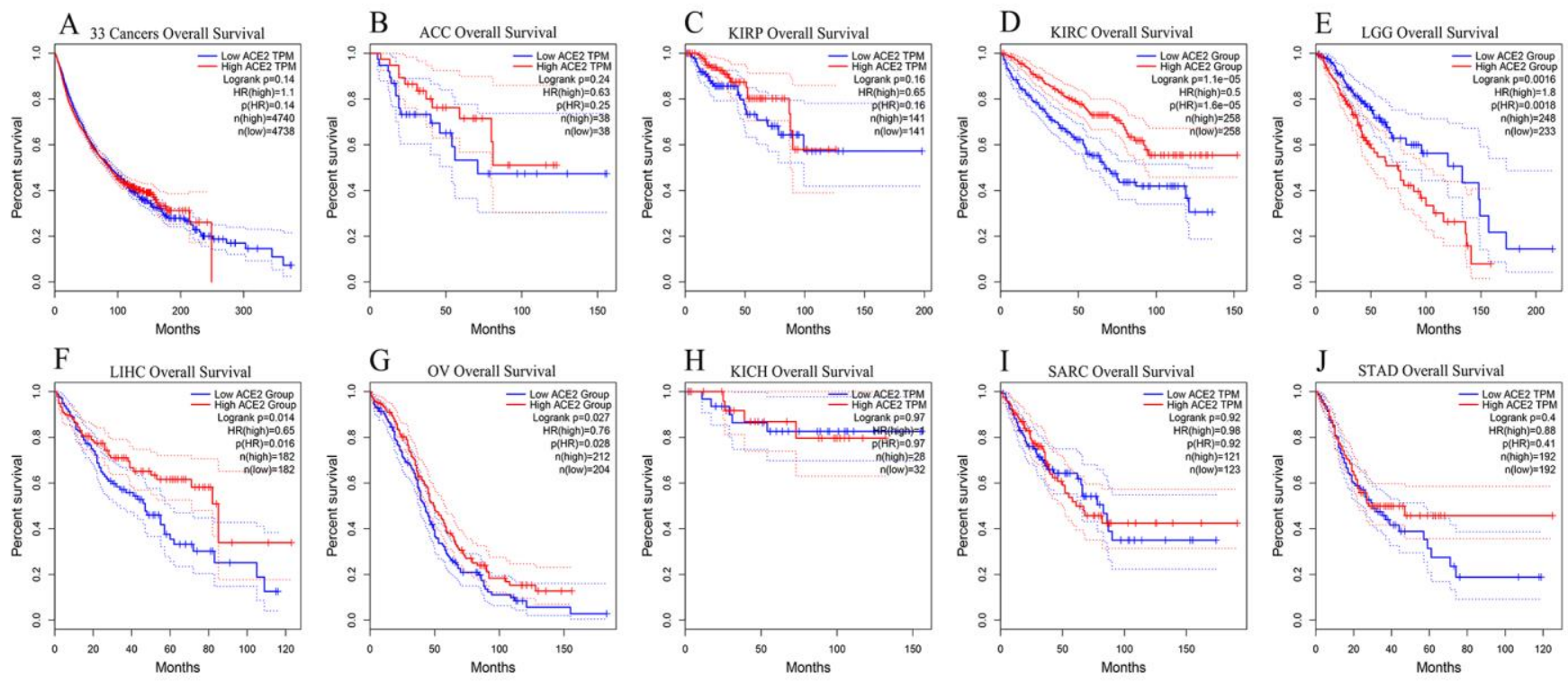


\section{Figure 5}

A

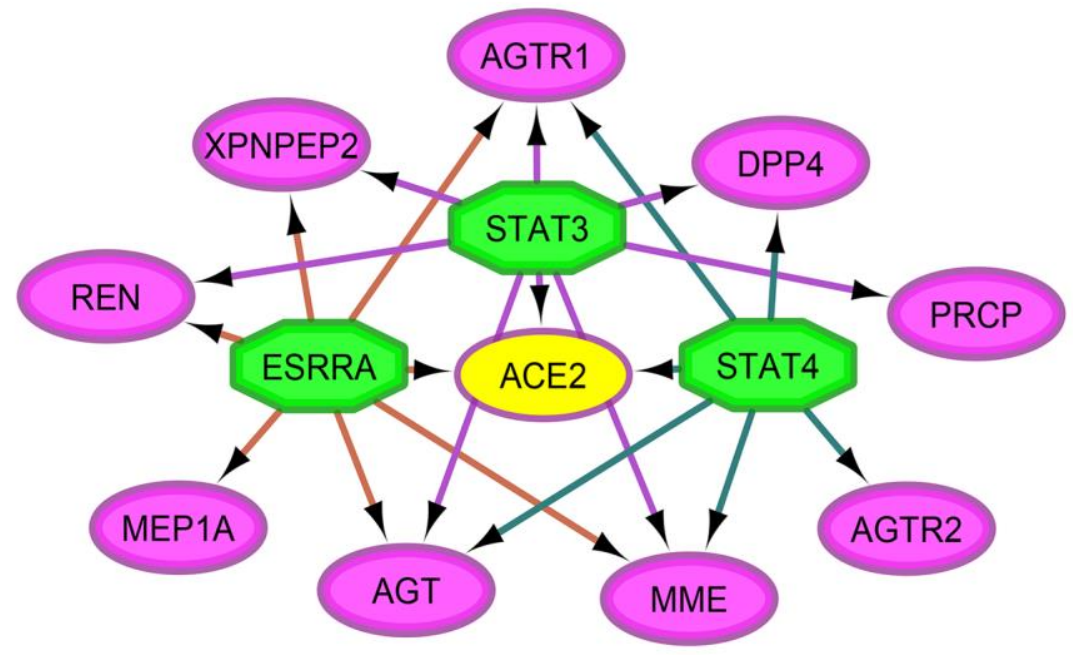

B

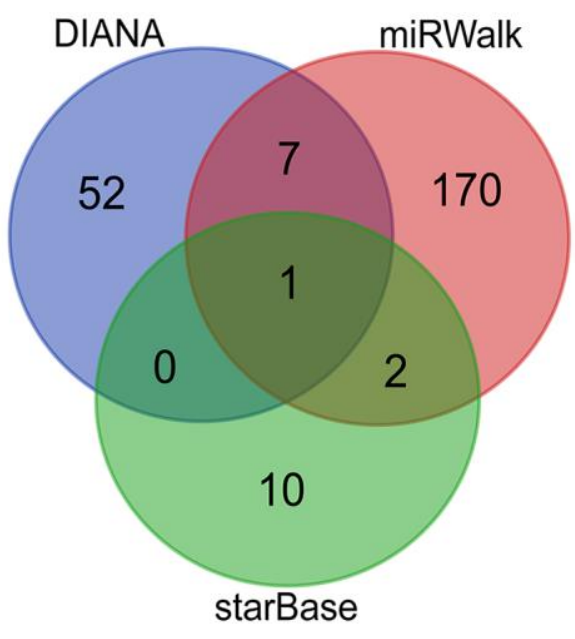


Table 1

GO Enrichment Term

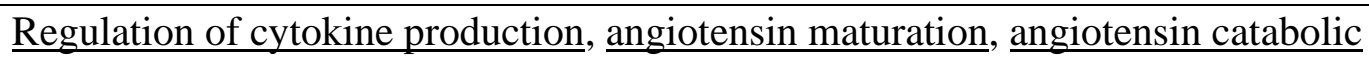
process in blood, angiotensin-mediated drinking behavior, regulation of systemic $\underline{\text { arterial blood pressure by renin-angiotensin, tryptophan transport, regulation of }}$ vasoconstriction, receptor biosynthetic process, $\underline{\text { regulation of cell proliferation, }}$ Biological Process regulation of vasodilation, viral entry into host cell, receptor-mediated virion $\underline{\text { attachment to host cell, }}$ regulation of inflammatory response, positive regulation of cardiac muscle contraction, positive regulation of gap junction assembly, $\underline{\text { regulation of cardiac conduction, positive regulation of reactive oxygen species }}$ $\underline{\text { metabolic process }}$

Cellular Component Extracellular region, extracellular space, cytoplasm, plasma membrane, cell surface, integral component of membrane, membrane raft, extracellular exosome Virus receptor activity, glycoprotein binding, endopeptidase activity, Molecular Function carboxypeptidase activity, metallocarboxypeptidase activity, protein binding, metallopeptidase activity, peptidyl-dipeptidase activity, zinc ion binding

KEGG Pathway Renin-angiotensin system, protein digestion and absorption 
Table 2

\begin{tabular}{lccc}
\hline Drug & Interaction type & Database & FDA Approved \\
\hline Lisinopril & Inhibitor & DGIdb & Yes \\
Chemb1260273 & Inhibitor & DGIdb & - \\
Chemb1429844 & Inhibitor & DGIdb & - \\
Flutamide & N/A & DGIdb & - \\
Chloroquine & Modulator & DrugBank & Yes \\
Hydroxychloroquine & Modulator & DrugBank & Yes \\
ORE-1001 & Inhibitor & DrugBank & - \\
N-(2-Aminoethyl)-1-aziridineethanamine & Inhibitor & DrugBank & - \\
Ramipril & Inhibitor & DrugBank & Yes \\
\hline
\end{tabular}

